\title{
Mr. WATARU
}

Irma Arribas Pérez

Expresión Gráfica Arquitectónica

ETSAB, UPC

irleta@me.com

\section{RESUMEN}

Mr. Wataru es una propuesta pedagógica para la asignatura de Dibujo 1 de la ETSAB, que responde a la naturaleza del dibujar en el quehacer proyectual.

Mr. Wataru defiende la acción del dibujar como un desplazarse por lugares desconocidos, permitiendo mediante tanteos y gestos que el dibujo aloje el proyecto y a su vez lo configure.

La acción del dibujar por encima del dibujo y como habilitador del proyecto es una naturaleza intensa que merece ser planteada en los inicios de los estudios de arquitectura, con la intención de formar a expedicionarios que conquisten, dígale innovar.

Experimentar es la naturaleza de la acción del dibujar que a su vez es proyectar, y que de una manera reflexiva y crítica puede llevar a la innovación.

Palabras clave: dibujar, alojar, expedicionario, proyectar

\section{ABSTRACT}

Mr. Wataru is a pedagogical approach to the ETSAB Drawing 1 Course which reflects the nature of the projective drawing tasks.

Mr. Wataru advocates for the action of draw as a navigation through unfamiliar places, allowing scores and gestures where drawing hosts and configures the project.

The action of drawing over the drawing and as a project enabler is an intense nature that deserves to be raised in the early studies of architecture, with the intention of training explorers to conquer, say innovate.

Experience is the nature of the action of drawing which in turn is projecting, and a reflective and critical manner that can lead to innovation.

Key words: drawing, host, expeditionary, project 


\section{Mr. WATARU}

\section{$1.1 \quad$ Presentación}

Mr. Wataru es un personaje que existe en el movimiento. Es en la acción de desplazarse donde es visible y su presencia no es una manifestación de su propia identidad. Su aparición esta causada por una búsqueda y se manifiesta por alojar en su presencia los indicadores de un cuerpo en creación.

Wataru significa "navegar" en japonés y Mr. Wataru fue adoptado para encabezar una propuesta pedagógica dentro de la asignatura de Dibujo 1 del departamento EGA, ETSAB, UPC.

La propuesta de Mr. Wataru es trabajar con la creencia y el interés que radica en la acción del dibujar como impulsor de un desplazamiento, una navegación, propio del proyectar y por otro lado, de la acción del dibujar como aquello que permite el "dejar aparecer" el proyecto, donde el "dibujo" no interesa tanto como la "acción del dibujar".

\section{$1.2 \quad$ Aparición}

Mr. Wataru, pretende investigar sobre el dibujo en el ámbito de la arquitectura y en relación al proceso proyectual, intentado crear en el aula un lugar de experimentación para la investigación del terreno del dibujar, investigación que mantengo desde las línea: "EDPA: La expresión del proyecto de arquitectura. Análisis y evolución"-ETSAB, UPC y "DIDI: Didáctica del Diseño"-EINA, UAB.

La innovación cabe ser entendida desde un proceso previo de investigación y experimentación que practica de la tolerancia y la complicidad de otros puntos de vista, para poder dejar aparecer, desde un pensamiento reflexivo y crítico otras manifestaciones, circuitos necesarios en el espacio/tiempo sobre el que nos establecemos.

Mr. Wataru no ha podido manifestarse en las aulas del 2013/2014, y muy tímidamente pudo aparecer en años anteriores.

Los intereses de Mr. Wataru, llevan tiempo siendo investigados en varios frentes y puesto en práctica en muchos otros.

Las dificultades de manifestarse en el aula, responden en parte a la escasa presencia de ejercer desde la experimentación los campos de investigación que permiten llegar a la innovación, al margen de mostrar las durezas de un sistema educativo en las escuelas de arquitectura que desde la teoría queda evidenciada su necesaria mutabilidad, como todo cuerpo, pero que desde la práctica el moldeo es difícil y costoso, seguramente porque los verdaderos intereses no son los de cuestionar el cuerpo, sino otros. 


\section{Miss. NOZOMI}

\subsection{Presentación}

Miss. Nozomi es el personaje elegido para aproximar la figura de Mr. Wataru. Nozomi significa esperanza en japonés.

Miss. Nozomi: "Conozco a Mr. Wataru desde hace mucho tiempo. Siempre ha sido una figura intrépida y aventurera, inquieta y provocadora, pero puedo decir que sus movimientos no son acciones simples, forman parte de una búsqueda crítica y reflexiva que atiende a necesidades reales.

Yo siempre he tenido mucha esperanza en sus movimientos, aun a ciegas; en sus palabras, aun en silencio. Sus intenciones son honestas, directas, estratégicas, sin perder de vista el objetivo: la naturaleza del dibujar en el quehacer proyectual."

\section{EL VIAJE DE CHIHIRO}

Para abordar el tema de la naturaleza del dibujar en el quehacer proyectual, se aportan algunas consideraciones sobre el ejercicio proyectual.

\subsection{Naturaleza de un Proyecto}

\subsubsection{Acción}

"Proyectar designa lanzar, dirigir hacia adelante, trazar o proponer un plan y los medios para la ejecución de una cosa, etc. El proyecto es el efecto del proyectar y significa el designio de efectuar algo. La noción de proyecto es tratada en varias filosofías y en las teorías del comportamiento y la acción. (...) interesa el proyecto como una irrealidad anticipada a la que se entrega el control de la conducta." (Segui, 1996)

Proyectar supone generar un marco, un ecosistema, unas condiciones para el "dejar aparecer" el proyecto. Esta atmósfera, no esta preestablecida en forma, pero si que puede ser definida a partir de la descripción de estructuras habilitadoras, instrumentos, acciones, gestos, circunstancias que abren el imaginario.

\subsubsection{Intención}

"El proyecto no comienza siendo una sólida imagen, sino un simple impulso que cobra su identidad en su propio desarrollo". (Eissenman)

Tras el impulso, la segunda intención es la de descodificar todo el complejo que se va elaborando, desarrollando, para que a través de la acción de descodificación se deje aparecer el proyecto.

Codificar un proyecto, en cambio, "disuelve la poética, identificándose en su totalidad con el procedimiento que simplemente la verifica; parte del proyecto pero, a propósito, no lo realiza, se limita a codificarlo como proyecto."

(Seguí, 1996)

La acción de codificar crea unas reglas internas, un código, motivo, que entrelaza unos agentes con un discurso único, unidireccional, cerrado. Esta naturaleza no pertenece a la acción proyectual. 


\subsubsection{Ejecutivo-ejecución}

Un proyecto ejecutivo "concibe la obra como idealmente hecha y, sucesivamente, dispone el plan de las diversas fases ejecutivas -el proyecto-, que tiene una función puramente instrumental, porque la obra está ya visualmente (imaginativamente) dada". (Argan, 1969)

La naturaleza del proyecto es mantenerse en ejecución: "el proyecto se construyen paso a paso y lo que cuenta es sólo la coherencia y el método que guía el movimiento."

\subsubsection{Condiciones}

En este apartado se explorarán las condiciones que diferencian "proyectar" de "proyecto". Dos realidades por separado, condiciones y ámbitos.

$>$ abierto/cerrado

Mientras que la acción de proyectar esta abierta, es "buscadora", "exploradora" de nuevos territorios, el proyecto es una cosa cerrada, o al menos en pausa.

\section{$>$ horizontal/vertical}

Mientras que el proyecto está alojado en una estructura de archivo vertical, en cajones organizados por códigos de año, tipología, etc, el proyectar pertenece a una condición de presencia horizontal, donde los cruces y las conmutaciones funcionan a un mismo plano, todas vistas, todas expuestas en la mesa de juego.

> escenografía/aparador

Proyectar es construir escenografías en donde la finalidad es que en ella sucedan cosas. No interesa tanto la definición ni la construcción de esta, sino las acciones que en ella se suceden. Un proyecto debe construir escenografías para alojar en las acciones que allí se produzcan los parámetros, los movimientos, la estructura del proyecto.

El proyecto es en cambio un aparador/vitrina. Una naturaleza muerta, capaz de poder ser activada de nuevo por el uso, reinterpretación, o operación de taxidermia.

$>$ laberinto/camino

El proyecto no podrá surgir de la acción de desplazarse por un camino.

La condición de camino lleva asociada la de un destino, un fin, un lugar concreto, una llegada. Por otro lado el tiempo empleado esta cuantificado, medido, constatado y premiada la agilidad de distancias máximas vs. tiempos cortos.

La condición de camino, lleva asociado la posibilidad de desviaciones, derivaciones, pero bajo la elección de estos ramales vuelve a replicarse la importancia de vencer al tiempo o vencer un mejor logro, un mejor fin, un mejor destino.

Proyectar supone adentrarse en un laberinto. Bajo esta simple acción existen dos encubiertas.

La primera hace referencia a que "Lo último que uno sabe es por dónde empezar". (Blas Pascal, 1623-1662).

La condición de adentrarse en un laberinto es mas atemorizadora que la de adentrase en un camino. El problema de partida no debiera existir, ya que el camino no debiera estar considerado y la naturaleza de la acción de proyectar debiera asumirse de forma mas relajada, sin miedos por que no hay motivo para ello. 
Lo importante es formar a exploradores, no a personas dominadas por el miedo que buscan la eficacia desde aquellos aspectos directos y de manejo fácil, despistando el verdadero interés de un viaje.

La segunda hace referencia al deslizarse por el recorrido interno, desconocido, indescifrable.

Lo importante para afrontar esta acción es el sujeto y su preparación para poder sumergirse en el entorno laberíntico que responde a la naturaleza de cada proyecto.

\section{Laberinto "Univiario"}

Solo existe una salida.

El recorrido es simple, una cuerda enrollada con sus dos cabos, entras por uno y sales por otro.

El problema no es si vas a salir, sino si vas a salir vivo.

No se toman decisiones, solo hay una vía posible.

\section{Laberinto "Manierista"}

Estructura de árbol, frondoso, con infinitas ramificaciones.

La estructura hace que tengamos que pisar sobre nuestros pasos erróneos en las decisiones. La condición de errar permanentemente se puede apoderar de la persona.

Solo nuestras mejores herramientas como la inteligencia, la memoria o en su defecto la fortuna puede ayudarnos a salir.

Puede haber monstruo dentro o no.

Laberinto "Rizoma" (red infinita)

Un estructura de conexiones en red que no tienen fin. No se puede reducir ni a lo "uno" ni a lo "múltiple". No tiene ni principio ni fin, solo un medio que crece y desborda.

El monstruo es el propio laberinto.

No existe ninguna lógica que lo gobierne. No hay salida.

\subsubsection{Condiciones}

Proyectar no tiene forma, es una acción.

Dibujar es dejar huellas, testimonios, registros que alojan parta del proyecto, pero el proyecto no cobra forma a través de la recolección de estos registros, a modo de sumatorio, de gran caja donde guardar, clasificar, etc. El proyecto es un dejar ir apareciendo, a lo largo de un tiempo empleado en donde la acción de proyectar no recoge los "mejores instantes" y los cataloga, sino que cuenta con la herencia, memoria, yuxtaposición, superposición. La metáfora nos puede llevar a asimilarlo a una torre de babel, donde el cuerpo se consolida por las convivencias del proceso.

\subsection{La condición del proyectar dibujando}

\subsubsection{Proyectar dibujando}

Proyectar dibujando es un movimiento que se ejerce desde la acción del dibujar, no como vehículo que ayuda a desplazarse dentro del proyecto, sino como acción que aloja en si mismo el proyecto y lo configura.

La cuestión estriba en diferenciar los comportamientos que hay entre el dibujo como instrumento de descubrimiento y navegación dentro del terreno del 
proyecto, (dibujo como herramienta, ágil, compensada, oportuna, delicada); frente al dibujo como alojante del proyecto, en donde el terreno del proyecto no es ajeno, no es un escenario/lugar a descubrir, sino que está por crear.

En el primer caso el dibujo es un servicio y el proyecto ya esta hecho.

En el segundo el proyecto se construye a través de la capacidad del dibujo.

En los dos casos se necesitan de un dibujo capaz, así como de un dibujante que quiera desplazarse, de varios escenarios en el dibujar; encontrar y narrar, pero la diferencia es grande y radica en el proyecto.

En el primero el proyecto esta hecho, por las tendencias, por la cultura, por lo hábitos, por la economía o por la política.

En el segundo el proyecto esta por hacer. Seguramente es mas interesante, ya que puede atender a todos los factores del contexto bajo un mirada interna, no impuesta, no heredada, mas libre, mas capaz.

\subsubsection{Formas de navegación}

Atendiendo al proyectar dibujando como acción configural, el dibujar se mueve entre dos culturas, separadas según el estado de navegación, imaginario/real, búsqueda/relato.

$>$ Navegación imaginaria

Estado: Procesual

Tiempo: Vivo

Capacidad: Alojar el proyecto

Forma: $\quad$ Sin forma, signos sin significado

Contenido: Registran acciones. Huellas. No representar

Dibujo: $\quad$ como metáfora, como gesto

Dibujar como acción metafórica, de búsqueda y exploración, como apertura no de la forma sino del cuerpo como gesto, potencia, posibilidad, fuerza, acción, no artefacto

Percepción: Invisible, táctil

$>$ Navegación real

Estado: Descriptivo

Tiempo: Muerto

Capacidad: Alojar figuras que representan y narran los acontecimientos de la navegación imaginaria

Forma: $\quad$ Con forma, signos con significado

Contenido: Representativo

Dibujo: $\quad$ como imitación, como técnica de representación

Dibujar: $\quad$ No se considera la acción

Percepción: Visible

La navegación imaginaria que ejerce el dibujo se basa en un desplazamiento de costumbres, hábitos, y pensamiento para dejar paso al sueño de la razón y descubrir monstruos.

\subsubsection{Acción}

$>$ lanzar

El proyecto necesita de estrategias imaginarias que le permitan lanzar, dirigir hacia adelante, trazar o proponer un plan. 
Dentro de estas estrategias, que atañen al imaginario, podríamos estructurar dos grupos:

grupo A: Aproximan entornos

Aquellas que contextualizan un ámbito lejano, des-localizado, extranjero con la intención de acercar una situación que cuestione desde los límites, desde la ausencia, la presencia del proyecto.

El uso de metáforas, usuarios extremos o la ficción, son estrategias hábiles en los inicios de un proyecto de diseño.

METAFORAS. Alojante de un contexto, un entorno dilatado.

USUARIO EXTREMO. Como figura extrañada del contexto cotidiano.

FICCIÓN. Usar la ficción como campo de simulación.

Grupo B: Buscan entornos

Aquellas que buscan a través de técnicas imaginaras, investigar y aproximarse a estados lejanos, configurando una red de instantes interrumpidos, búsquedas des localizadas, capaces de alojar el proyecto.

Se podría definir la acción del dibujar como un impulso empírico de apertura y exploración, fuera de toda compromiso con el representar para atender al estado naciente de un proyecto de diseño.

ACCIÓN DEL DIBUJAR

ACCIÓN DE ESPECULAR

La naturaleza que une a la acción del lanzar como accionador de un proyecto es la necesidad de imaginar, no la de un imaginario ni la de una imagen. Se trata de aproximar una irrealidad.

La imagen tiene la capacidad de turbar el cuerpo. Las imágenes, como decía Aristóteles, son lo que hace posible que el recuerdo se convierta en memoria, en afección en sensación y pensamiento.

En este sentido, el comportamiento del dibujar ha de atender a factores como son el tantear/sin pensar/con gestos/desafío/"inquietud perpetua"/"conocimiento a través de la prueba" (G.Didi)/digestión interna/no visible-si táctil.

Dibujar supone un ir y venir, sin miedo, no saber ni importar los efectos secundarios, supone atender a los imprevistos, supone entregar el control a la acción/reacción. En la acción, el tiempo se mide no de forma cuantitativa sino cualitativa y en es fuera del pensamiento donde reside la probabilidad y la posibilidad. La inquietud llama a desafiar las leyes, las reglas, las normas y a cuestionar los cuerpo, las formas, las acciones.

\subsubsection{Intención}

Dibujar pretende anticipar una irrealidad desconocida, en donde reside un mundo de posibles.

Proyectar dibujando pretende navegar en una búsqueda a través de crear escenarios que condensen datos, que muestren datos, que visibilicen datos, que confronten datos, fuera de los datos.

\subsubsection{Re-acción}

Proyectar, pasa por considerar tres reacciones frente a la acción del dibujar:

> "Sorprenderse" por las figuras graficas que aparecen. 
Se hace visible no solo lo capaz del dibujo sino la vitalidad del proyectista, la mirada atenta del que tras accionar es capaz de interpretar y sorprenderse tras la crítica.

> "Interpretar", leer, verbalizar, escuchar, escribir, juzgar lo trazado y los proceso de formación.

Tras la acción, se ha de recoger, traducir a escritura, palabras, crítica lo que alli subyace, el proyecto alojado.

Solo desde este lado consciente y de traducción a otro estado, de la grafía visual a la grafía sonora, es posible la interpretación y con ello que el ejercicio proyectual continúe.

$>$ "Dialogar", reconocer el lenguaje y gestos como un reclamo.

En el ejercicio de dibujar reside la capacidad de dialogar no solo como instrumento de comunicación externa sino de construcción. Solo un dibujo consciente y atento es capaz de contener datos que pueden ser cuestionados y bajo este acto de diálogo continuar dibujando para construir en base a lo puesto en crisis y asi, dialogando, moverse.

\subsubsection{Condición}

$>$ Sin lugar

Dibujar no tiene un lugar, se sitúa en un espacio intermedio.

La superficie que lo recoge es un terreno infinito de acción en donde la atención no es del dibujo a la superficie sino de la superficie a la acción del dibujar.

Por otro lado, el dibujo aloja el proyecto, y él mismo se convierte en proyecto, por lo que no puede "estar", solo "dejar aparecer".

$>$ Sin movimiento (con tiempo, en pausa no inmóvil)

Un dibujo no posee movimiento, pero puede ser un condensador de tiempo, puede accionar una pausa no inmóvil.

Danzar por fantasmata es un termino usado en danza para describir aquellos momentos, en donde el bailarín no se mueve, siendo capaz de catalizar, condensar en ese instante de quietud todo la coreografía, un momento de tensión máxima, un cuerpo que aloja toda una información, un instante que acumula todo un tiempo.

"El verdadero lugar del bailarín no está en el cuerpo y en sus movimientos, sino en la imagen (...) como pausa no inmóvil, sino cargada, al mismo tiempo, de memoria y de energía dinámica". "Como en el "danzar por fantasmata" de Doménico de Piacenza, la vida de las imágenes no consiste en la simple movilidad ni en la sucesiva recuperación del movimiento, sino una pausa cargada de tensiones entre ambas". Giorgio Agamben.

El dibujo posee esta condición de condensación, como una cámara tensa, cargada.

$>$ Sin forma

"Drawing is the opening of a form". Jean Luc Nancy (7)

Para Nancy, dibujar es la apertura de una forma que requiere de un trazar a través de gestos, potencia, posibilidad, fuerza. Pero mas que la forma que traza, 
la huella manifestada, interesa el sujeto y su forma de moverse, de trazar en la superficie. Se trataría mas de la apertura de un cuerpo, no de una forma.

Interesa la no forma, la, lo, el sin-forma.

"La Niña de los Peines, tuvo que desgarrar su voz porque sabía que la estaba oyendo gente exquisita que no pedí formas, sino tuétano de formas, música pura con el cuerpo sucinto para poderse mantener en el aire". García Lorca (8)

Una forma contiene, aloja, ordena, bajo una cuerpo objetual/figural finito, acabado. Podría decirse que su naturaleza es mas la de un aparador que la de un escenario.

Proyectar necesita mas escenarios y menos aparadores. Dibujar debe atender a construir escenarios mas que a registrar formas capaces de alojar, guardar ordenadamente la naturaleza del proyecto.

"Perder el sentido de la forma es perder tu camino, perderte, perder la cabeza. Los diseñadores, como guardianes de la forma, son guardianes de la seguridad psicológica. Son un servicio de seguridad. Agentes de vigilancia, en contra de lo que Lynch describe como locura, confusión. Disgusto, frustración, alienación, ansiedad, miedo, angustia, terror y pánico que aflora al perderse". Wigley, Mark (9)

El dibujo no está relacionado con un afuera, no se parece a nada exterior, sino a algo interior, al acontecimiento y al sujeto que lo han creado.

\subsubsection{Accionador}

$>$ Viajero

El viajero ansia su llegada al punto de destino. Inicia el viaje por un fin, atendiendo a los acontecimientos de forma altamente resolutiva, inodora, incolora, para poder llegar lo antes posible y con el mismo cuerpo con el que salió.

\section{$>$ Expedicionario}

El explorador desconoce su punto de llegada y como el viajero, desconoce los acontecimientos que puedan surgir.

Un de las grandes diferencias es que mientras el viajero evita o hace desaparecer los acontecimientos, el explorador los utiliza para trazar el rumbo de su viaje.

Mantener la escucha en el proceso proyectual, los oídos abiertos, el cuerpo tonificado, las neuronas oxigenadas, es absolutamente necesario dado que el proyecto no esta al final del camino, en esos momentos, solo está el proyectar.

Dibujar es una "terapia" configurar para experimentar con uno mismo (Sloderdijk), de naturaleza intercultural pero que necesita de la interpelación del lenguaje verbal para poder ser interpretada.

\subsection{Una pausa}

La acción del dibujar forma parte del ejercicio proyectual, en donde mas que hablar de dos realidades, proyecto-dibujo, podríamos decir que hay solo una, la de un proceso. 
Es necesario aclarar la naturaleza del dibujo por su complicidad en el proceso proyectual, desde la citada navegación imaginaria hasta la navegación real, el dibujo que acerca el imaginario y el que cuenta lo sucedido. En todo caso no interesa establecer la naturaleza del dibujar en relación al dibujo como producto, ni a las técnicas que le dan forma, ni al pensamiento que lo dirige, ni a las destrezas que no permiten abrir otros lenguajes.

Mr. Wataru aparece en el primer año de arquitectura para incidir en la idea de la acción del dibujar tal y como ha sido expuesta, para no crear confusiones, para poder comenzar con la acción proyectual desde el principio, para hacer del aprendizaje una aventura, formar a expedicionarios, ansiar el movimiento, no tener miedo, atender a las necesidades de los estudiantes que entran a la arquitectura como espacio de un instante, espacio celeste, espacio imaginario, espacio blanco, espacio nocivo, espacio del interior, la conquista del espacio.

Partir del buscar para el encontrar, sin plano ni hoja de ruta, formando desde lo reflexivo y lo crítico en el material que se va generando, agudizando miradas, tacto, oído y olfato.

Se ha de tener una necesidad de moverse, no una imposición. Interesa el como desplazarse, no adelantando la meta, sino atendiendo a los parámetros del recorrido. Los movimientos generados son difíciles de retener en la mente, si "se olvidan no se pueden volver a dibujar. Todas sus caras o lados tienen igual importancia. No tiene relaciones jerárquicas predeterminadas. El frente y los laterales dependerán del observador y no del objeto. (...). Los instrumentos que se manejan para formalizar un objeto arquitectónico no son ya, en principio, los propios de la composición arquitectónica. La arquitectura se producirá desde nuevos puntos de partida en los que la forma ya no tendrá valor en si". (15)

Solo hay que atender a un proyecto de arquitectura como una negociación donde "ajustar por vías aparentes e interesadas un convenio entre todos los materiales que conformarán la arquitectura". (15)

Interesa pues formar a negociadores, expedicionarios, aventureros, arquitectos. 


\section{BIBLIOGRAFIA}

(1) Janes, J. "El origen de la conciencia en la ruptura de la mente bicameral". Ed. Fondo de cultura económica, 1987

(2) Didi-Huberman, G. "Arde la imagen". Mexico, Ed. Oceano, 2012

(3) Fallman, D. Art: "The Interaction Design Research Triangle of Design Practice, Design Studies, and Design Exploration", Design Issues (summer 2008, Volume 24, Number 3, p.4-18)

(4) Batlle, G. "El limite de lo útil". Madrid. Ed. Losada, 2005

(5) Segui, J. "Dibujar Proyectar III. Madrid: Instituto Juan de Herreros ETSAM, 2003

(6) Arendt, H. "labor, trabajo, acción" en "De la Historia a la Acción" Barcelona, Ed. Pahidos Iberica, 1995

(7) Nancy, JL. "Le Plaisir au dessin". Paris: Galilée, 2009

(8) García Lorca, F. "Juego y teoría del duende" 1933. En "Obras completas". Madrid, Ed. Aguilar, 1986

(9) Wigley, M. "Lost in space", en Haan, Jasper; Graafland, Arie (Ed.), The Critical Landscape, 010 Publisher, Rotterdam, 1995.

(9) Agamben, G. "Ninfa". Valencia, Ed. Pre-textos, 2010

(10) Derrida, J, "Memories of the blind: the selft-portrait and other ruins". USA. The University of Chicago Press, 1993

(11) Kerényi, K. "En el laberinto". El árbol del paraíso 48. Madrid: Ed. Siruela

(12) Cosme Ibañez, N. "Aproximación al laberinto. Una panorámica". Tesis doctoral. Departamento de Dibujo, Facultad Bellas Artes "Alonso Cano", Universidad de Granada, 2010.

(13) Pérec, G. "Especies de espacios". Edición española propiedad de Literatura y Ciencia, S.L. Primera Edición, Abril 1999.

(14) Kosma, A. "Proyectar dibujando: Una aproximación fenomenológica al estado naciente del proyecto: Un estudio entre dos culturas arquitectónicas". Tesis doctoral. Departamento de ideación gráfica, ETSAM, UPM, 2014

(15) Soriano, F. "Sin tesis". Barcelona, Ed Gustavo Gili, 2004 